\title{
MODIFIED WIENER-HOPF EQUATION IN IDENTIFICATION PROBLEMS
}

\author{
Valeriy Nikolaevich Afanasyev' ${ }^{1}$, Dmitry Vitalievich Titov² \\ ${ }^{1}$ Institute of Electronics and Mathematics, Moscow, Russian Federation \\ ${ }^{2}$ Southwest State University, Kursk, Russian Federation
}

The method of adaptive filtration in the tasks of flow settings of cosmic radiation recovery by the measured data for the use in cosmic transport systems with a long life cycle was shown in the article. Optimization mathematic model and algorithm of non-stationary control systems, in which the measurement is made against the background of the noise, are described. Parametric optimization algorithms are done by the use of modified Wiener-Hopf equation and function of sensitivity.

Key words: Algorithm, Optimization, Condition, Operator, Wiener-Hopf

\section{INTRODUCTION}

\section{THE PROBLEM OF THE NON-STATIONARY OBJECT IDENTIFICATION}

\section{Optimality conditions}

It is proposed to consider the construction of the basic design of non-stationary control systems optimization algorithms, whose state measurement is performed against a background of interference [1, 2, 3]. The concept developed in the process of optimization algorithms construction can be used to solve a wide range of problems - from the identification system construction and the solution of filtering non-stationary processes tasks to the construction of parametric control algorithms for non-stationary objects.

For definiteness, let the object be described by an ordinary differential equation

$$
\begin{aligned}
& \frac{d}{d t} x(t)=f(x, u, w, t), \\
& x\left(t_{0}\right)=x_{0},
\end{aligned}
$$

and measuring its state

$$
y(t)=C_{x}(t)+n(t)
$$

where $x \in \mathrm{R}^{n}, y \in \mathrm{R}^{\mathrm{m}}, n \geq m, \mathrm{w}(\mathrm{t})$ and $n(t)$ - Gaussian noise; where as $E[w(t)]=0, E[n(t)]=0$,

$\mathrm{E}$ - sign of expectation and

$E\left[\left(\begin{array}{c}w(t) \\ n(t)\end{array}\right)\left(w^{T}(\tau) n^{T}(\tau)\right)\right]=\left(\begin{array}{ll}W(t) & \Pi(t) \\ \Pi^{T}(t) & N(t)\end{array}\right) \delta(t-\tau)$,

$\delta(t-\tau)$ - Dirac function, ${ }^{\top}-$ transpose sign.

Note that change in time in the vector-function $f(x, u, w, t)$ occurs as a result of the fact that the parameters of this vector-function are under the influence of external perturbations. i.e. $f(x, u, w, t)=f(x, u, w, \eta(t))$, where $\eta(t)$ - the object parameters that vary according to an unknown law, and, whereas

$$
\begin{aligned}
& \|\eta(t)\| \leq \mathrm{H}=\text { const }>0, \\
& \left|\frac{d}{d t} \eta(t)\right| \leq \Lambda=\text { const }>0, \quad t \geq t_{0} .
\end{aligned}
$$

Let

$$
\varepsilon(t)=\mathrm{L}(t) x(t)-\Psi(t) \hat{x}(t)
$$

where $\hat{x}(t) \in \mathrm{R}^{q}$ - process estimation - solution of the differential equation.

$$
\begin{aligned}
& \frac{d}{d t} \hat{x}(t)=f_{m}(y, u, \alpha(t)), \\
& \hat{x}\left(t_{0}\right)=\hat{x}_{0},
\end{aligned}
$$

$\varepsilon(t) \in \mathrm{R}^{k}$ - observation error, $L \epsilon R, \psi \epsilon R$ - linear operators that transform $\mathrm{R}^{n} \rightarrow \mathrm{R}^{k}$ and $\mathrm{R}^{q} \rightarrow \mathrm{R}^{k}$ correspondently, $\alpha \in R^{\prime}$ - vector of model optimization parameters. Suppose that the function twice differentiable with respect to $\alpha(t)$.

It should be noted that the step of model structure choice is extremely responsible. The appropriateness, applicability and effectiveness of the evaluation design essentially depend on the reliability with which the mathematical model describes the actual situation (object, measurements, and external parametric disturbances). In most practical problems, a complete, accurate model is not available at all, and its construction has great difficulties, and therefore the problem of evaluation design for the measured process $y(t)$ must be solved with incomplete knowledge of the model. Even more complicated is the problem, when the noise $w(t)$ and $n(t)$ and/or the object parameters (2.1) change in an uncontrolled manner.

In addition, stochastic object state determination described by nonlinear differential equations, from measurements of its phase components against a noise background, requires the solutions of nonlinear differential equations. Moreover, the exact construction, for example, of a nonlinear filter is impossible and, what is 
very important, the estimation of the accuracy of approximation for a suboptimal realization of a nonlinear filter is either difficult or impossible.

Quadratic functionals are often used while synthesis of optimal systems.

$$
J(x(\cdot), \hat{x}(\cdot))=E\|L(t) x(t)-\Psi(t) \hat{x}(t)\|^{2} .
$$

Before finding the general minimum condition for a functional of the form (2.5), we note that a sufficiently large number of control objects models can be described with the help of systems of linear differential equations with incomplete information on parameters and the state vector. For such models, it is fair

$$
\hat{x}(t)=\mathfrak{I}(\alpha(t), t) y(t)
$$

where $\mathfrak{I}(\alpha(t), t) \in \mathrm{R}$ - the space of linear operators with optimization parameters $\alpha(t)$.

In the general case, restrictions can be imposed on the linear operator $\mathfrak{I}(\alpha(t), \tau)$ structure and parameters, i.e. operator $\mathfrak{I}(\alpha(t), t) \in \mathrm{R}_{1}$, where $\mathrm{R}_{1} \subset \mathrm{R}$, has an involuntary (predetermined) structure.

We show that the optimal operator

$\mathfrak{I}^{0}(\alpha(t), t)=\mathfrak{I}\left(\alpha^{0}(t), t\right) \in \mathrm{R}_{1}$

where $\alpha^{\circ}(t)$ - the operator optimization parameters $\mathfrak{I}(\alpha(t), t) \in \mathrm{R}$, for which $\hat{x}(t)$ the minimum gives the functional (2.5), satisfies condition (1.7) $\mathrm{N}(t) \neq 0$

$$
E\left[\left\{L(t) x(t)-\Psi(t) \Im\left(\alpha^{0}(t), t\right) y(t)\right\}^{T}\{\boldsymbol{N}(t), y(t)\}\right]=0 \text {, }
$$

where $\mathfrak{\aleph}(t)$ - zero linear operator.

For the Gaussian processes and linear operators condition (1.6) is a necessary and sufficient condition for the minimum of the functional (1.5) and has the form of the Wiener-Hopf equation. To prove this proposition, we introduce

$$
\mathfrak{I}(\alpha(t), t)=\mathfrak{I}\left(\alpha^{0}(t), t\right)+\lambda \mathfrak{\aleph}(t)
$$

Here $\mathfrak{I}(\alpha(t), \tau) \in \mathrm{R}_{1}, \mathfrak{N} \in \mathrm{R}_{1}$ - linear nonzero operator $\lambda$ - weight coefficient.

Taking into consideration that the operator $\mathfrak{I}(\alpha(t), \tau)$ coincides with the optimal operator $\mathfrak{I}\left(\alpha^{0}(t), \tau\right)$ only when $\lambda=0$, we will get

$$
\frac{\partial}{\partial \lambda} J(\ldots \lambda) \text { when } \lambda=0
$$

i.e.

$\frac{\partial}{\partial \lambda} E\left\|L(t) x(t)-" \Psi^{\prime \prime}(t)\left[" \Im^{\prime \prime}(\alpha(t), t)+" \lambda \boldsymbol{N}^{\prime \prime}(t)\right] y(t)\right\|^{2}=0$, where $\lambda=0$.

As a result, we obtain an expression that coincides with (1.6).

A condition of the form (1.7) can be obtained if we investigate the functional when $\alpha(t)=\alpha^{\circ}(t)$.

The optimality conditions will have the form:

$$
\begin{aligned}
& {\left[\frac{\partial}{\partial \alpha(t)} J(x(t), \hat{x}(t))\right]^{T}=E\left[-\left\{\frac{\partial \Psi(t) \hat{x}(t)}{\partial \alpha(t)}\right\}^{T}\{L(t) x(t)-\Psi(t) \hat{x}(t)\}\right]=0,} \\
& \frac{\partial}{\partial \alpha(t)} J(x(t), \hat{x}(t))=E\left[\left\{\frac{\partial \Psi(t) \hat{x}(t)}{\partial \alpha(t)}\right\}^{T} \frac{\partial \Psi(t) \hat{x}(t)}{\partial \alpha(t)}\right]>0, i, j=1, \ldots, l, \text { where } \alpha(t)=\alpha^{0}(t)
\end{aligned}
$$

Here $\left\{\frac{\partial \Psi(t) \hat{x}(t)}{\partial \alpha(t)}\right\}^{\mathrm{T}}=\aleph(t)$ - leaner operator.

If the operators and its model space is linear, condition (1.6) is a necessary and sufficient condition for the minimum of the functional (1.5). Generally, when the space of the object operators and its model is arbitrary, condition (1.6) is sufficient and additional restrictions on the type of these operators are required to obtain the necessary conditions.

Thus, equation (1.6) for linear operators can have only one solution. The following issue remains open - is there a solution at all. However, the question of the existence of equation (1.6) solution is found automatically in all cases when it is possible to find the solution to this equation.

Theorem 1. Let an object be described by an ordinary differential equation

$$
\begin{aligned}
& \frac{d}{d t} x(t)=f(x, u, w, t), \\
& x\left(t_{0}\right)=x_{0},
\end{aligned}
$$

and measurements of its state $y(t)=C x(t)+n(t)$, where $x \in \mathrm{R}^{n}, y \in \mathrm{R}^{m}, n \geq m, w(t)$ and $n(t)$ - centered Gaussian noise. Then the optimal operator $\mathfrak{I}^{0}(\alpha(t), t)=\mathfrak{I}\left(\alpha^{0}(t), t\right) \in \mathrm{R}$ where $\mathrm{R}$ the space of linear operators, $\alpha^{\circ}(\mathrm{t})$ - the values of the operator optimization parameter $\mathfrak{I}(\alpha(t), t) \mathrm{s}$, under which $\hat{x}(t)$ gives the minimum to the functional $J(x, \hat{x})=E\|L(t) x(t)-\Psi(t) \hat{x}(t)\|^{2}$, satisfies condition $E\left[\left\{L(t) x(t)-\Psi(t) \mathfrak{T}\left(a^{0}(t), t\right) y(t)\right\}^{T}\left\{\boldsymbol{N}^{\prime}(t) y(t)\right\}\right]=0$, N'(t) $\neq 0$

\section{The basic optimization algorithms design in identification problems}

We make a number of assumptions about the object. Let the object be described by a nonlinear differential equation

$$
\frac{d}{d t} x(t)=f(x, u, w, t), x\left(t_{0}\right)=x_{0}
$$

The vector-function $f(x, u, w, t)$ contains parameters $\eta(t)$ $\epsilon \mathrm{R}_{\eta}$ that vary under the influence of external perturbations, i.e. $f(x, u, w, t)=f(x, u, w, \eta(t))$, and allows differentiation with respect to the set of variables the required number of times. The object model is a model described by the equation: 


$$
\begin{aligned}
& \frac{d}{d t} \hat{x}(t)=f_{m}(y, u, \alpha(\mathrm{t})), \\
& \hat{x}\left(t_{0}\right)=\hat{x}_{0} .
\end{aligned}
$$

The identification quality criterion in this problem has the form

$$
J(\varepsilon(\cdot))=E[F(\varepsilon(t, \alpha, " \eta "))] .
$$

Here $F(\varepsilon(t, \alpha, \eta))=\varepsilon^{\top}(t, \alpha, \eta) \varepsilon(t, \alpha, \eta)-$ loss function.

$$
\frac{d}{d t} J(\varepsilon(t, \alpha, \eta))=\frac{\partial}{\partial t} J(\varepsilon(\cdot))+\frac{\partial}{\partial \alpha(t)} J(\varepsilon(\cdot)) \frac{d}{d t} \alpha(t)+\frac{\partial}{\partial \eta(t)} J(\varepsilon(\cdot)) \frac{d}{d t} \eta(t) \leq 0 .
$$

Taking into account (1.11) and the fact that the functional (1.9) does not depend explicitly on t, we obtain

$$
\begin{aligned}
& \frac{d}{d t} J(\varepsilon(t, \alpha, \eta))=-\left\{E\left[\left\{\frac{\partial F(\varepsilon(t, \alpha, \eta))}{\partial \alpha(t)}\right\}\right]\right\} E\left[\left\{\frac{\partial(\varepsilon(t, \alpha, \eta))}{\partial \alpha(t)}\right\}^{T}\right]+ \\
& +\left\{E\left[\left\{\frac{\partial F(\varepsilon(t, \alpha, \eta))}{\partial \eta(t)}\right\}\right]\right\} \frac{d}{d t} \eta(t) \leq 0 .
\end{aligned}
$$

Obviously, this condition will be satisfied if the speed of the adjustment of the model parameters satisfies the condition

$$
\left\|E\left[\frac{\partial F(\varepsilon(t, \alpha, \eta))}{\partial \alpha(t)}\right]\right\|^{2}>\left|\left\{E\left[\frac{\partial(\varepsilon(t, \alpha, \eta))}{\partial \eta(t)}\right]\right\} \frac{d}{d t} \eta(t)\right| .
$$

Thus, the fulfillment of condition (1.12) guarantees the successful changes "tracking" in the object parameters with the chosen algorithms for changing the model paue asymptotically. for the identifiability of the non-stationary system. tion algorithm has the form (1.11)

$$
\begin{gathered}
\frac{d}{d t} \alpha=E\left[\left\{\frac{\partial \Psi(t) \hat{x}(t)}{\partial \alpha(t)}\right\}^{T}\{L(t) x(t)-\Psi(t) \hat{x}(t)\}\right], \\
\alpha\left(t_{0}\right)=\alpha_{0} .
\end{gathered}
$$

$$
\begin{gathered}
\frac{d}{d t} \alpha(t)=-E\left[\left\{\frac{\partial(\varepsilon(t, \alpha, n))}{\partial \alpha(t)}\right\}^{T}\right], \\
\alpha\left(t_{0}\right)=\alpha_{0} .
\end{gathered}
$$

One can use the following algorithm [4] in problems of non-stationary object identification by its mathematical model with parametric optimization

Let us find a condition in which the necessary properties to the optimization process provide the algorithms of the form (1.11). This condition has the form

rameters (1.9). This ensures "transfer" of the quality functional from any peripheral values to its minimum val-

The assumption of observability of the object and the inequality (1.12) form necessary and sufficient conditions

For quadratic functionals of the form (1.5), the optimiza-

As for the value of the quality functional (1.5), adopted when implementing an identification system with optimization algorithms of the type (1.11),there is the ratio

$$
\begin{aligned}
& E\left\|L(t) x(t)-\Psi(t) \Im^{\prime \prime}\left(a^{0}(t), \tau\right) y(t)\right\|^{2} \leq E\left\|L(t) x(t)-" \Psi^{\prime \prime}(t) " \Im^{\prime \prime}(a(t), \tau) y(t)\right\|^{2} \\
& \text { or } J\left(x, \hat{x}^{0}\right) \leq J(x, \hat{x}) .
\end{aligned}
$$

\section{THE MODIFIED WIENER-HOPF EQUATION IN PROBLEMS OF FILTERING NON-STATIONARY PROCESSES}

We consider the problem of vector of a random Gaussian Markov process estimation construction in the space from measurements of a part of its coordinates produced against a noise background. Despite longitudinal research in this field, interest in such tasks is not weakened, which, for example, is confirmed by a number of reports made at [5].

Let a useful process be specified as the result of passing nonstationary Gaussian white noise through a linear dynamical system

$$
\begin{aligned}
& \frac{d}{d t} x(t)=A(t) x(t)+B(t) w(t), \\
& x\left(t_{0}\right)=x_{0} .
\end{aligned}
$$

The measured process is specified

$$
y(t)=C x(t)+n(t)
$$

In (2.1) and (2.2) $x \in \mathrm{R}^{n}, y \in \mathrm{R}^{m}, w \in \mathrm{R}^{r}, n \in \mathrm{R}^{m}$. Nonstationary processes $w(t)$ and $n(t)$ - white Gaussian centered nois- es with intensities $W(t)$ and $N(t)$. The pair $\langle A, C\rangle$ are observed.

There is no loss of generality in supposing that noises $w(t)$ and $n(t)$ are not correlated and

$$
\left[x\left(t_{0}\right) w^{\top}(t)\right]=0, M\left[x\left(t_{0}\right) n^{\top}(t)\right]=0 .
$$

Defined problem of construction of the failure in the least- deviation sense

$$
J(\varepsilon)=E\left[\varepsilon^{T}(t) \varepsilon(t)\right]
$$

where

$$
\varepsilon(t)=x(t)-\hat{x}(t)
$$

$\hat{x}(t)$ - efficiency process assessment, known as optimum filtering problem.

Kalman-Bucy filter [1] is written as

$$
\begin{gathered}
\frac{d}{d t} \hat{x}(t)=A(t) \hat{x}(t)+K(t)[y(t)-C \hat{x}(t)], \\
\hat{x}(t)=\overline{x_{0}}=E\left[x\left(t_{0}\right)\right], \\
K(t)=P(t) C E^{-1}(t)
\end{gathered}
$$

where $P(t)=E\left[\varepsilon(t) \varepsilon^{T}(t)\right]$ - deviation error matrix, that is solution of the Riccati equation type (2.7).

Integral of a differential equation in this case will be filtering error (2.8). 


$$
\begin{gathered}
\frac{d}{d t} P(t)=A(t) P(t)+P(t) A^{T}(t)-P(t) C^{T} N^{-1}(t) C P(t)+B(t) W(t) B^{T}(t), \\
P\left(t_{0}\right)=E\left[\left(\varepsilon_{0}(t) \varepsilon_{0}^{T}(t)\right)\right] . \\
\frac{d}{d t} \varepsilon(t)=[A(t)-K(t) C] \varepsilon(t)+B(t) w(t)-K(t) n(t), \\
\varepsilon\left(t_{0}\right)=x_{0}-\bar{x}_{0} .
\end{gathered}
$$

Note that optimal filter (2.5) has a structure of the efficiency process generator (2.1) and matrixes $A(t), B(t)$, $W(t)$ and $E(t)$ completely specify its parameters. In this respect filter can be thought of as an efficiency process model.

Implementation of the filter as (2.5)-(2.7) is impossible if matrixes $A(t), B(t), W(t)$ and $E(t)$ vary in time over unknown law.

A tentative assumption should be made to construct a filter with parametric optimization. We assume that

$$
A(t)=A+a(t), B(t)=B+b(t) .
$$

Then we can rewrite equation (3.1) as

$$
\begin{aligned}
& \frac{d}{d t} x(t)=[A+a(t)] x(t)+[B+b(t)] w^{*}(t), \\
& x\left(t_{0}\right)=x_{0},
\end{aligned}
$$

where $a(t), b(t)$ - matrixes of perturbation parameters, $w^{*}(t)$ - stationary white noise with the intensity $W$ (non-stationary nature of the process $w(t)$ is "taken" into changing of the parameters of the matrix $b(t)$ ).

We assume that matrix of non-stationary white noise intensities can be conceived of as $E(t)=E+\delta(t)$.

We also assume that uncertainty has interval character, i.e. the following inequations subsist:

$$
\begin{gathered}
\underline{a}_{i j} \leq a_{i j}(t) \leq \bar{a}_{i j}, \underline{b}_{i k} \leq b_{i k}(t) \leq \bar{b}_{i k}, \underline{\delta}_{k l} \leq \delta_{k l}(t) \leq \bar{\delta}_{k l}, \\
i, j=\overline{1, n}, k, l=\overline{1, r}, t \geq t_{0}
\end{gathered}
$$

(underline - minimum, overline - maximum).

The main structure of the filter will be constructed the following way

$$
\frac{d}{d t} \hat{x}(t)=[A+\phi(t)] \hat{x}(t)+\left[K^{*}+k(t)\right][y(t)-C \hat{x}(t)] \text {, }
$$$$
\hat{x}\left(t_{0}\right)=\bar{x}_{0} \text {, }
$$

where matrix $K^{*}$ estimate as solution of the equation:

$$
K^{*}=P^{*} C N^{-1} \text {. }
$$

where $P^{*}$ positive definite matrix of Riccati algebraic equation solution

$A P^{*}(t)+P^{*}(t) A^{T}-P^{*}(t) C^{T} N^{-1} C P^{*}(t)+B W^{*} B^{T}=0$.

Solutions (2.13) and (2.12) are realized at the non-stationary filter design stage.
Thus, filter is constructed with an accuracy to parameter point of matrixes $\phi(t)$ and $k(t)$, which optimize filter operation for the purpose of functional (2.3) while getting $E\left[\{x(t)-\hat{x}(t)\}^{T}(L(t) y(t)\}\right]=\operatorname{tr} E[\{x(t)-$ $\left.-\hat{x}(t)\}\{L(t) y(t)\}^{T}\right]=0$.

appropriate information.

Optimizing algorithm realization. In the case under study necessary and sufficient condition of the functional minimum (2.3) is described by the Wiener-Hopf equation:

Here a nonzero linear operator $L(t)$ transforms a vector $y \in R^{m}$ into a vector $z \in R^{m}$. Thus, the optimality condition (2.14) is achieved in the space of vectors $\varepsilon(\mathrm{t})$ and, when $\varepsilon(t) \perp\{\mathrm{L}(t) y(t)\}$. The condition (2.14) of the optimality of the estimation (2.11), as noted above, must be satisfied for any non-zero linear operator $L(t) \in R$.

Taking into account the method of optimization algorithms forming proposed in Section 1 of the article (for example, algorithm (1.12)), the condition (2.14) should be the base of the algorithms for parametric optimization (identification) of the filter (2.11). However, estimated algorithms would be unrealizable, because of the need for having the process available.

To construct realizable algorithms for filter optimization (2.11), we introduce the functional

$J_{1}\left(e(t), e\left(t_{1}\right)\right)=\operatorname{tr} E\left[e^{T}(t) e\left(t_{1}\right)\right]$,

where:

$e(t)=y(t)-C \hat{x}(t), \quad e\left(t_{1}\right)=y(t+\gamma)-C \hat{x}(t+\gamma), \quad \gamma \neq 0$

time shifting.

Definition 1: Two functionals introduced to evaluate the solution of the filtration problem are equivalent if they reach their minima for the same values of the filter parameters. In this case, the values of the minima can be different.

For small values of the shift, in comparison with the dynamics of the model (2.11), the functional (2.15) is equivalent in the above sense to the original functional (2.3). In view of (2.14), we write the expression for the functional (2.15)

$$
J_{1}\left(e(t), e\left(t_{1}\right)\right)=\operatorname{tr}\left\{C E\left[e^{T}(t) e^{T}\left(t_{1}\right)\right] C^{T}+C E\left[e(t) n^{T}\left(t_{1}\right)\right]+E\left[n(t) \varepsilon^{T}\left(t_{1}\right)\right] C+E\left[n(t) n^{T}\left(t_{1}\right)\right]\right\}
$$


To find the values of the expression $E\left[e(t) n^{T}\left(t_{1}\right)\right]$, we of the differential equation (2.8). Then assume that $\Phi(t, T)$ is a fundamental matrix of solutions

$$
E\left[\varepsilon(t) n^{T}\left(t_{1}\right)\right]=E\left[\Phi\left(t, t_{0}\right) \varepsilon(t) n^{T}\left(t_{1}\right)+\int_{t_{0}}^{t} \Phi(t, \tau)[B(\tau) w(\tau)-K(\tau) n(\tau)] n^{T}\left(t_{1}\right) d \tau\right]=0
$$

due to the fact that $E\left[\varepsilon(t) n^{T}\left(t_{1}\right)\right]=0, E\left[w(t) n^{T}\left(t_{1}\right)\right]=0$

and $E\left[n(t) n^{T}\left(t_{1}\right)\right]=0$ where $t_{1}=t+\gamma, y \neq 0$

Thus

$$
J_{1}\left(e(t), e\left(t_{1}\right)\right)=\operatorname{trEC}\left[\varepsilon(t) \varepsilon^{T}\left(t_{1}\right)\right] C^{T}, t_{1}=t+\gamma, \gamma \neq 0
$$

Comparing (2.17) and (2.3), we can conclude that the functional (2.17) reaches a minimum for the same values of the filter parameters as the functional (2.3). In this sense, these functionals are equivalent; i.e.
$J_{1}\left(e(t), e\left(t_{1}\right)\right), J(\varepsilon)$.

It is easy to see that the necessary conditions for the minimum of the functional (2.17) have the form

$$
E\left[\left\{y\left(t_{1}\right)-C \hat{x}\left(t_{1}\right)\right\}^{T}\left\{L_{1}(t) y(t)\right\}\right]=E\left[\{y(t)-C \hat{x}(t)\}^{T}\left\{L_{1}\left(t_{1}\right) y\left(t_{1}\right)\right\}\right]=0, t_{1}=t+\gamma
$$

Here the operator $L_{1}(t)$ converts the vector $y \in R^{m}$ into a vector $z_{1} \in R^{m}$.

Definition 2: The necessary and sufficient condition

(3.18) for the minimum of the functional

$J_{1}\left(e(t), e\left(t_{1}\right)\right)=\operatorname{trCE}\left[\varepsilon(t) \varepsilon^{T}\left(t_{1}\right)\right] C^{T}, t_{1}=t+\gamma, \gamma \neq 0$, will be

called the modified Wiener-Hopf equation.
If, in (2.18), we substitute the expression for $y(t)(2.2)$, then it can be shown that condition (2.18) is necessary and sufficient for the minimum of both the functional (2.17) and the functional (2.3). Really,

$$
\operatorname{tr} E\left[\{y(t)-C \hat{x}(t)\}\left\{L_{1}\left(t_{1}\right) y\left(t_{1}\right)\right\}^{T}\right]=\operatorname{trCE}\left[\{x(t)-\hat{x}(t)\}\left\{L_{1}\left(t_{1}\right) y\left(t_{1}\right)\right\}^{T}\right]+\operatorname{tr} E\left[n(t)\left\{L_{1}\left(t_{1}\right) y\left(t_{1}\right)\right\}^{T}\right]
$$

due to the fact that $E\left[\hat{x}\left(t_{2}\right) n^{T}\left(t_{1}\right)\right]=0$ and

$E\left[n(t) n^{T}\left(t_{1}\right)\right]=0$, i.e.

$\operatorname{trE}\left[\{y(t)-C \hat{x}(t)\}\left\{L_{1}\left(t_{1}\right) y\left(t_{1}\right)\right\}^{T}\right]=\operatorname{trCE}\left[\{x(t)-\hat{x}(t)\}\left\{L_{1}\left(t_{1}\right) y\left(t_{1}\right)\right\}^{T}\right]=0$,

$L_{1}\left(t_{1}\right)=C L\left(t_{1}\right), t_{1}=t+\gamma$.

Theorem 2. For the system

$$
\begin{aligned}
& \frac{d}{d t} x(t)=A(t) x(t)+B(t) w(t), x\left(t_{0}\right)=x_{0}, \\
& y(t)=C x(t)+n(t),
\end{aligned}
$$

where $E\left[x\left(t_{0}\right) w^{T}(t)\right]=0, E\left[x\left(t_{0}\right) n^{T}(t)\right]=0, E\left[w(t) n^{T}(t)\right]=0$, functional

$J_{1}\left(e(t), e\left(t_{1}\right)\right)=E\left[e^{T}(t), e_{1}\left(t_{1}\right)\right], e(t)=y(t)-C \hat{x}(t), t_{1}=t+\gamma, \gamma \neq 0$,

the necessary and sufficient conditions for a minimum of which have the form

$E\left[\left\{y\left(t_{1}\right)-C \hat{x}\left(t_{1}\right)\right\}^{T}\left\{L_{1}(t) y(t)\right\}\right]=E\left[\{y(t)-C \hat{x}(t)\}^{T}\left\{L_{1}\left(t_{1}\right) y\left(t_{1}\right)\right\}\right]=0$, reaches a minimum under the same values of the filter parameters as the functional

$J(\varepsilon)=E\left[\varepsilon^{\top}(t) \varepsilon(t)\right]$

the necessary and sufficient conditions for a minimum of which have the form

$E\{x(t)-\hat{x}(t)\}^{\top}\{L(t) y(t)\}=0$.

These functionals are equivalent in the sense of Definition 1.

\section{ALGORITHMS OF PARAMETRIC OPTIMIZATION}

Equation (1.12) is based on the design of filter optimization algorithms (1.12). We will carry out some additional constructions. We arrange the vectors $\phi^{*}(t)$ and $k^{*}(t)$ the dimension of both $n^{2} \times 1$ and $\left(n^{*} m\right) \times 1$ from the matrix elements $\phi(t)$ and $k(t)$, respectively.

Taking into account what was said in Subsection 1.2, we will represent the design of optimization algorithms in the form of:

$$
\begin{gathered}
\frac{d}{d t} \phi^{*}(t)=E\left[\left\{\Pi_{\phi}(t) y(t)\right\}\left\{y\left(t_{1}\right)-C \hat{x}\left(t_{1}\right)\right\}\right], \\
\phi^{*}\left(t_{0}\right)=\phi_{0}^{*}, \\
\frac{d}{d t} k^{*}(t)=E\left[\left\{\Pi_{k}(t) y(t)\right\}\left\{y\left(t_{1}\right)-C \hat{x}\left(t_{1}\right)\right\}\right], \\
k^{*}\left(t_{0}\right)=k_{0}^{*},
\end{gathered}
$$

here $\Pi_{\phi}(t)$ and $\Pi_{k}(t)$ - linear operators that transform $\mathrm{m}$-dimensional vector into dimension matrices $\left(n^{*} n\right) \times m$ and $\left(n^{*} m\right) \times m$ and, respectively.

We first consider the case when the parameters of the matrices $a$ and $b$ are constant but not known. When choosing operators $\Pi_{\phi}(t)$ and $\Pi_{k}(t)$ we require that the optimization process, i.e. the transfer of the quality functional from its peripheral values to a minimum, had the property of asymptotics. This means that the following condition must be met $\frac{d}{d t} J_{1}\left(y(t), \hat{x}\left(t_{1}\right)\right) \leq 0$, i.e..

$$
\frac{\partial J_{1}\left(y(t), \hat{x}\left(t_{1}\right)\right)}{\partial t}+\frac{\partial J_{1}\left(y(t), \hat{x}\left(t_{1}\right)\right)}{\partial \phi^{*}(t)} \frac{d \phi^{*}(t)}{d t}+\frac{\partial J_{1}\left(y(t), \hat{x}\left(t_{1}\right)\right)}{\partial k^{*}(t)} \frac{d k^{*}(t)}{d t} \leq 0
$$


Since the functional in the case under consideration does not depend explicitly on $t$, and $y(t)$ does not depend on the optimization parameters $\varphi^{*}(t)$ and $k^{*}(t)$, condition (3.3), taking into account (3.15), can be rewritten in the following way:

$$
\begin{gathered}
E\left[-\left\{\frac{\partial \hat{x}(t)}{\partial \phi^{*}\left(t_{0}\right)}\right\}^{T}\left\{y\left(t_{1}\right)-C \hat{x}\left(t_{1}\right)\right\}\right]^{T} E\left[\left\{\Pi_{\phi}(t) y(t)\right\}\left\{y\left(t_{1}\right)-C \hat{x}\left(t_{1}\right)\right\}\right]+ \\
E\left[-\left\{\frac{\partial \hat{x}(t)}{\partial k^{*}(t)}\right\}^{T}\left\{y\left(t_{1}\right)-C \hat{x}\left(t_{1}\right)\right\}\right]^{T} E\left[\left\{\Pi_{k}(t) y(t)\right\}\left\{y\left(t_{1}\right)-C \hat{x}\left(t_{1}\right)\right\}\right] \leq 0
\end{gathered}
$$

It is easy to see $\Pi_{\varphi}(t)$ that the operators $\Pi_{k}(t)$ and choice or appointment in the form

$\Pi_{\phi}(t) y(t)=\left\{\frac{\partial C \hat{x}(t)}{\partial \phi^{*}(t)}\right\}^{\mathrm{T}}, \Pi_{k}(t) y(t)=\left\{\frac{\partial C \hat{x}(t)}{\partial k^{*}(t)}\right\}^{\mathrm{T}}$

provide optimization process with the asymptotic properties. The algorithms (3.1) and (3.2), taking (4.5) into account, take the form:

$$
\begin{gathered}
\frac{d}{d t} \phi^{*}(t)=E\left[\left\{\frac{\partial C \hat{x}(t)}{\partial \phi^{*}\left(t_{0}\right)}\right\}^{T}\left\{y\left(t_{1}\right)-C \hat{x}\left(t_{1}\right)\right\}\right], \\
\phi^{*}(t)=\phi_{0}^{*}, \\
\frac{d}{d t} k^{*}(t)=E\left[\left\{\frac{\partial C \hat{x}(t)}{\partial k^{*}(t)}\right\}^{T}\left\{y\left(t_{1}\right)-C \hat{x}\left(t_{1}\right)\right\}\right], \\
k^{*}\left(t_{0}\right)=k_{0}^{*} .
\end{gathered}
$$

$$
E\left[\left\{\frac{\partial^{2} C \hat{x}(t)}{\partial \phi_{i}^{*}(t) \partial \phi_{j}^{*}(t)}\right\}^{T}\left\{y\left(t_{1}\right)-C \hat{x}\left(t_{1}\right)\right\}\right]=0,
$$

The positive definiteness of the second derivatives demonstrates the possibility of attaining the minimum value of the quality functional $J_{1}\left(y(t), \hat{x}\left(t_{1}\right)\right.$, for unknown, but constant values of the parameters of the matrices $a, b$ and in the process equation (2.10) when using algorithms of the form (3.6).

Consider the case when $a(t)$ and $b(t)$. We arrange the vectors $a(t)$ and $b(t)$ that have dimensions $n^{2} \times 1$ and $\left(n^{*} m\right) \times 1$ from matrices elements $a(t)$ and $b(t)$ relatively.
In the algorithms (3.6), the first factors are the sensitivity functions.

The conditions determining the optimal values of the parameters of the matrices $\phi(t)$ and $k(t)$ will be written on condition $\phi(t)=\phi^{0} ; k(t)=k^{0}$ in the following way:

$$
\begin{aligned}
& \frac{\partial}{\partial \phi^{*}(t)} J_{1}\left(y(t), \hat{x}\left(t_{1}\right)\right)=2 E\left[\left\{-\frac{\partial C \hat{x}(t)}{\partial \phi^{*}(t)}\right\}^{T}\left\{y\left(t_{1}\right)-C \hat{x}\left(t_{1}\right)\right\}\right]=0, \\
& \frac{\partial}{\partial k^{*}(t)} J_{1}\left(y(t), \hat{x}\left(t_{1}\right)\right)=2 E\left[\left\{-\frac{\partial C \hat{x}(t)}{\partial k^{*}(t)}\right\}^{T}\left\{y\left(t_{1}\right)-C \hat{x}\left(t_{1}\right)\right\}\right]=0 . \\
& \frac{\partial}{\partial \phi_{i}^{*}(t) \partial \phi_{j}^{*}\left(t_{1}\right)} J_{1}\left(y(t), \hat{x}\left(t_{1}\right)\right)=2 E\left[\left\{\frac{\partial C \hat{x}(t)}{\partial \phi_{i}^{*}(t)}\right\}^{T} \frac{\partial C \hat{x}\left(t_{1}\right)}{\partial \phi_{j}^{*}\left(t_{1}\right)}\right]>0, \\
& \frac{\partial}{\partial k_{i}^{*}(t) \partial k_{n}^{*}\left(t_{1}\right)} J_{1}\left(y(t), \hat{x}\left(t_{1}\right)\right)=2 E\left[\left\{\frac{\partial C \hat{x}(t)}{\partial k_{i}^{*}(t)}\right\}^{T} \frac{\partial C \hat{x}\left(t_{1}\right)}{\partial k_{n}^{*}\left(t_{1}\right)}\right]>0, \\
& i, j=\overline{1, n^{2}} ; i, h=\overline{1, n \cdot m}
\end{aligned}
$$

because

$E\left[\left\{\frac{\partial^{2} C \hat{x}(t)}{\partial \mathcal{K}_{i}^{*}(t) \partial \mathcal{K}_{h}^{*}(t)}\right\}^{T}\left\{y\left(t_{1}\right)-C \hat{x}\left(t_{1}\right)\right\}\right]=0$.

The condition $\frac{d}{d t} J_{1}\left(y(t), \hat{x}\left(t_{1}\right)\right) \leq 0$ in this case is written in the following form

$$
\begin{aligned}
& \frac{\partial J_{1}\left(y(t), \hat{x}\left(t_{1}\right)\right)}{\partial t}+\frac{\partial J_{1}\left(y(t), \hat{x}\left(t_{1}\right)\right)}{\partial a^{*}(t)} \frac{d a^{*}(t)}{d t}+\frac{\partial J_{1}\left(y(t), \hat{x}\left(t_{1}\right)\right)}{\partial b^{*}(t)} \frac{d b^{*}(t)}{d t}+ \\
& +\frac{\partial J_{1}\left(y(t), \hat{x}\left(t_{1}\right)\right)}{\partial \phi^{*}(t)} \frac{d \phi^{*}(t)}{d t}+\frac{\partial J_{1}\left(y(t), \hat{x}\left(t_{1}\right)\right)}{\partial k^{*}(t)} \frac{d k^{*}(t)}{d t} \leq 0 .
\end{aligned}
$$

Taking into account the choice of the algorithms (3.6), we will get the following:

$$
\begin{aligned}
& \frac{d}{d t} J_{1}\left(y(t), \hat{x}\left(t_{1}\right)\right)=\frac{d}{d t} E\left[e^{T}\left(t_{1}\right) e(t)\right]=2 E\left[e^{T}\left(t_{1}\right)\left(\left\{\frac{d y(t)}{d a^{*}(t)}\right\} \frac{d a^{*}(t)}{d t}+\left\{\frac{d y(t)}{d b^{*}(t)}\right\} \frac{d b^{*}(t)}{d t}\right)\right]- \\
& -2 E\left[e^{T}\left(t_{1}\right)\left(\left\{\frac{d C \hat{x}(t)}{d \phi^{*}(t)}\right\} \frac{d \phi^{*}(t)}{d t}+\left\{\frac{d C \hat{x}(t)}{d k^{*}(t)}\right\} \frac{d k^{*}(t)}{d t}\right)\right] \leq 0
\end{aligned}
$$

wherefrom, taking into account (4.6):

$$
E\left[e^{T}\left(t_{1}\right)\left(\left\{\frac{d y(t)}{d a^{*}(t)}\right\} \frac{d a^{*}(t)}{d t}+\left\{\frac{d y(t)}{d b^{*}(t)}\right\} \frac{d b^{*}(t)}{d t}\right)\right]-E\left[e^{T}\left(t_{1}\right)\left(\left\{\frac{d C \hat{x}(t)}{d \phi^{*}(t)}\right\}\left\{\frac{d C \hat{x}(t)}{d \phi^{*}(t)}\right\}^{T}+\left\{\frac{d C \hat{x}(t)}{d k^{*}(t)}\right\}\left\{\frac{d C \hat{x}(t)}{d k^{*}(t)}\right\}^{T}\right) e\left(t_{1}\right)\right] \leq 0,
$$

So, it gives us the condition for the algorithms of parametric optimization effectiveness (3.6):

$$
E\left[e^{T}\left(t_{1}\right)\left(\left\{\frac{d C \hat{x}(t)}{d \phi^{*}(t)}\right\}\left\{\frac{d C \hat{x}(t)}{d \phi^{*}(t)}\right\}^{T}+\left\{\frac{d C \hat{x}(t)}{d k^{*}(t)}\right\}\left\{\frac{d C \hat{x}(t)}{d k^{*}(t)}\right\}^{T}\right) e\left(t_{1}\right)\right]>\left|E\left[e^{T}\left(t_{1}\right)\left(\left\{\frac{d y(t)}{d a^{*}(t)}\right\} \frac{d a^{*}(t)}{d t}+\left\{\frac{d y(t)}{d b^{*}(t)}\right\} \frac{d b^{*}(t)}{d t}\right)\right]\right| .
$$


Thus, if this condition is fulfilled when the parameters of the process generator (2.10) and the filter parameters (2.10) are modified in accordance with the algorithms (3.6), the conditions for maintaining a suboptimal state are satisfied at least.

\section{CONCLUSION}

The algorithms for optimizing the observers of nonstationary processes with a quadratic functional of quality under the conditions of incomplete a priori information are presented and investigated in this paper. The basement of all the obtained algorithms is the modified Wiener-Hopf method. This method is a necessary and sufficient condition for the minimum of the auxiliary functional, which is equivalent to the given functional, but contains only the available information.

We have got the conditions for the successful systems optimization, written in the form of inequalities, which include the sensitivity of the functionals to changes in the perturbations and the response of the parameters extracted for parrying or / and compensating for these disturbances and their speed.

The method on the basis of which the algorithms for optimizing observers were developed can be used both for solving problems of filtering non-stationary processes and for constructing algorithms for parametric identification of non-stationary objects, and it means that the algorithm can be widely applied.

A lot of automation problems are solved by the use of robotic systems, in which accuracy is very important. Such systems are used in flexible manufacturing systems, space researches. One of the basic elements of robotic systems is an electric drive, which helps to decrease oscillations of the current stabilization and electromotor speed by the use of the above offered methods. The efficiency of the offered algorithm increases in 4 times.

Another example of the use of the modified Wiener-Hopf method is the movement control of the space satellite, which is stabilizing without external disturbance. Output disturbance amplitude is in 30 times less then input output disturbance.

\section{REFERENCES}

1. Kalman R., Busy R. New Results in Linear Filtering and Prediction Problems // Trans. ASME J. Basic Engineering. 1961. V. 83. No. 1. P.123-141.

2. Hassibi Babak, Sayed Ali N., Kailath Thomas. Indefinite-Quadratic Estimation and Control. Philadelphia, SIAM. 1999.

3. Khrustalev M.M., Khalina A.S. Optimal Controller synthesis for Linear Stochastic Systems with Incomplete Information Regarding the State. Necessary Conditions and Numerical Method. // Autom. Remote Control. 2014. V. 25. No. 11. P.1448-1463.

4. Afanasiev V.N., Control of undetermined dynamic objects. M.: Nayka, 2008, 210 P.

5. Cacace F., Conte F., Germani A. A new filtering approach for continuous-time linear systems with delayed measurements // Proc. 19th World Conf. IFAC. Cape Town, South Africa. 2014. P.3745-3750. 